\title{
Monkey brain cortex imaging by use of photoacoustic tomography
}

Xinmai Yang, Lihong V. Wang

Xinmai Yang, Lihong V. Wang, "Monkey brain cortex imaging by use of photoacoustic tomography," Proc. SPIE 6856, Photons Plus Ultrasound: Imaging and Sensing 2008: The Ninth Conference on Biomedical Thermoacoustics, Optoacoustics, and Acousto-optics, 685608 (28 February 2008); doi: 10.1117/12.762396

SPIE. Event: SPIE BiOS, 2008, San Jose, California, United States 


\title{
Monkey brain cortex imaging by use of photoacoustic tomography
}

\author{
Xinmai Yang and Lihong V. Wang ${ }^{*}$ \\ Optical Imaging Laboratory, Department of Biomedical Engineering, Washington University in St. \\ Louis, St. Louis, Missouri 63130
}

\begin{abstract}
Photoacoustic tomography (PAT) is adopted to image the brain cortex of monkeys through the intact scalp and skull ex vivo. The reconstructed PAT image shows the main structure of the blood vessels on the monkey brain cortex. For comparison, the brain cortex is imaged without the scalp then imaged again without the scalp and skull. Ultrasound attenuation through the skull is also measured at various angles of incidence to illuminate the effect of the incident angle. This study demonstrates that PAT of the brain cortex is capable of surviving the ultrasound signal attenuation and distortion caused by a considerably thick skull.
\end{abstract}

Keywords: photoacoustic tomography, image reconstruction, monkey brain

\section{INTRODUCTION}

High-resolution human brain imaging modalities currently being used in clinics include X-ray computerized tomography (CT) and magnetic resonance imaging (MRI). However, both CT and MRI are expensive. Furthermore, $\mathrm{X}$-ray CT involves exposure to ionizing radiation. In certain cases, ultrasound brain imaging is adopted ${ }^{1}$. For example, ultrasound imaging is an established pediatric brain imaging modality ${ }^{2}$ before the fontanelles are closed. After the closure of the fontanelles, the image quality degrades significantly because the skull severely attenuates and scatters ultrasonic waves. Transcranial ultrasonic brain imaging of adults is also limited by the inhomogeneous aberrating effect of the skull bone ${ }^{3-9}$. The skull bone induces both phase and amplitude aberrations, which need to be corrected. In order to improve transcranial ultrasonic brain imaging, various methods have been proposed, such as adaptive focusing ${ }^{10}$, time reversal ${ }^{11}$, dynamic focusing ${ }^{12}$, and the spatio temporal inverse filter (STIF) ${ }^{13}$. However the transcranial ultrasound imaging still suffers from poor resolution or poor contrast, and only a few structures can be identified.

Photoacoustic tomography (PAT) is a hybrid, non-ionizing imaging modality, which combines the resolution of ultrasound imaging with the contrast of optical imaging ${ }^{14}$. In PAT, pulsed laser energy is delivered into biological tissues. The subsequent ultrasound signal generated by the photoacoustic (PA) effect is detected and an image can be formed through a reconstruction algorithm. Compared with pure ultrasound imaging, the signal contrast in PAT is provided by optical contrast, which is $2-3$ orders of magnitude higher than the ultrasound contrast. PAT is particularly suitable for imaging blood vessels because of the high optical contrast between blood and other biological tissues. In addition, PAT detects ultrasound signals which are from a one-way propagation. Therefore less distortion is expected in transcranial photoacoustic signals and the images have a better chance to survive distortion than pure ultrasound images.

Brain cortex imaging, including structure, functional, and molecular imaging, by PAT has been successfully conducted on small animals ${ }^{15}$. However, the brain cortex imaging by the same modality on large animals has not been studied. Our group has reported monkey brain imaging using thermoacoustic tomography (TAT) ${ }^{16}$ and showed the feasibility of applying TAT on a thin skull brain. Compared with TAT, whose contrast is determined by water content of biological tissues, PAT provides stronger contrast, and is more suitable for vascular imaging.

* Contact Information: lhwang@biomed.wustl.edu

Photons Plus Ultrasound: Imaging and Sensing 2008: The Ninth Conference on Biomedical Thermoacoustics, Optoacoustics, and Acousto-optics, edited by Alexander A. Oraevsky, Lihong V. Wang, Proc. of SPIE Vol. 6856, 685608, (2008) - 1605-7422/08/\$18 - doi: 10.1117/12.762396 
We intend to extend the application of PAT to large animal brain cortex imaging in this study. Two monkey head samples were obtained from nation primate research centers, and we implemented our PAT system to image the brain cortex of these samples. Although the skulls of monkey heads used in our experiment are considerably thick $(\sim 2$ and $\sim 3$ $\mathrm{mm}$ ), the main structures of the monkey brain cortex can still be identified clearly in PAT images. This promising result shows the feasibility of large animal brain cortex imaging using PAT. The outcome of this study may provide a useful diagnostic tool for human brain cortex imaging in the future

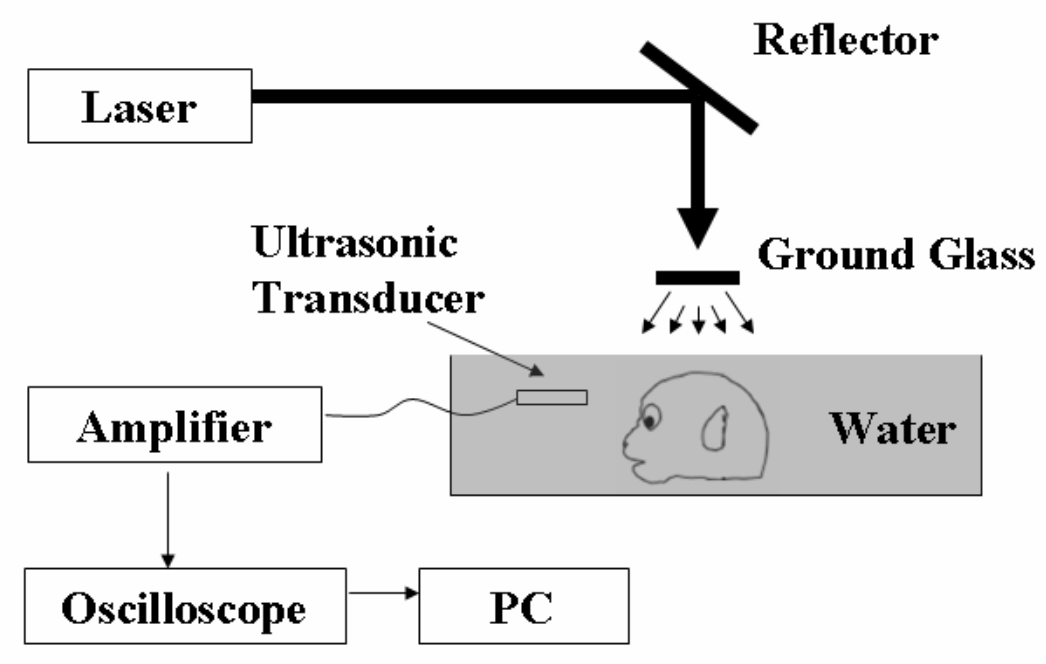

Figure 1 Diagram of experimental setup

\section{METHODS AND MATERIALS}

The experimental setup for this study is shown in Fig. 1. A Q-switched Nd:YAG laser (pulse width $<15$ ns) (LS2137/2, Symphotic TII Co. Camarillo, CA), operating at $1064 \mathrm{~nm}$ or $532 \mathrm{~nm}$ with a pulse repetition rate of $10 \mathrm{~Hz}$, was employed as the irradiation source. A monkey head sample was irradiated from the top, and PA signals were detected on the orthogonal plane by an ultrasound transducer, and then amplified by a 50-dB amplifier (5072 PR, Panametrics, Waltham, MA). Next, the signals were directed to a digital oscilloscope (TDS5054, Tektronix, Beaverton, OR) and collected through a PC.

The early work by Fry et al ${ }^{17}$ showed that ultrasound signals propagating through a skull suffer from less distortion at frequencies below $1 \mathrm{MHz}$. Therefore, a flat transducer (V303, Panametrics, Waltham, MA) with a center frequency of 1 $\mathrm{MHz}$ and $60 \%$ bandwidth was used in our experiment. The active element size of the transducer is $12 \mathrm{~mm}$. 
Before the PAT of a monkey brains, we first tested if there was enough energy left after laser light passes through a monkey skull. A carbon rod cross was placed in the water tank and the laser light was illuminating from top. The laser light was attenuated by a 5-mm thick skull bone before it hit the carbon rods. In this experiment, there was a free path for photoacoustic signals to propagate from the target to the detector. In the next experiment, we tested if thusgenerated PA signals can propagate through the skull bone. We attached the carbon rod cross to the inner surface of the skull so that the generated PA signals will pass through the skull before they arrives at the ultrasound detector, whereas the laser light was also attenuated.

A fresh head sample of a 20-month old rhesus monkey was obtained from California Primate Research Center. The head sample was immersed in $10 \%$ formalin solution immediately after the tissue was harvested. The first PAT experiment was performed on the 5th day after the animal was sacrificed. Before the experiment, the hairs on the head were removed to reduce the potential attenuation to acoustic waves. The dimensions of the head in axial view are about $9 \times 7 \mathrm{~cm}$. The estimated skull thickness is $\sim 2 \mathrm{~mm}$.

Before the experiment, the ultrasound transducer was carefully aligned to point to the scanning center. The head sample was then put into the water tank and immersed in water. The location of the water tank was adjusted so that the sample was at the central region of scanning. During the data acquisition, the ultrasound detector scanned the sample circularly for 240 steps with a scanning radius of $10 \mathrm{~cm}$. A simple back-projection algorithm ${ }^{18}$ was employed to reconstruct an image. The laser wavelength used in all experiments was $1064 \mathrm{~nm}$ unless otherwise indicated.

After the initial experiment, the skull was opened and the monkey brain was exposed. We found that the brain tissue still looked fresh when the skull was opened. This is because it is hard for formalin to penetrate the skull. We also found that blood still remained in the major blood vessels, which gave us the optical contrast.

The PAT imaging of the exposed brain was conducted next. Before imaging the exposed brain, the skull bone was put back on the monkey brain to keep the brain in its original shape. Then the head sample was immersed in the $10 \%$ formalin solution. After the brain was fixed by formalin, the skull bone was removed and the shape of the brain remained unchanged. The formalin solution also helped to solidify blood inside the blood vessels so that blood will not diffuse into water when the brain was put into water. After this process, the exposed brain was imaged.

Next, to highlight the effect of the skull bone on PAT, we put the skull bone back on the exposed brain, and another PAT image was obtained. Compared with the original intact head sample, the scalp and dura mater were removed. The relative location between the skull and blood vessels might also change, and it will have an effect on the incident angle of PA signals when these signals propagate to the skull inner surface. We also repeated this process with $532 \mathrm{~nm}$ laser wavelength to compare the effect of the laser wavelength.

Besides the young rhesus monkey head sample, another fresh head sample of an 8-year old pigtail monkey was obtained from Washington Primate Research Center, and imaged with PAT. Before imaging, this head sample was processed with the similar procedure as the previous rhesus monkey head. The dimensions of this head sample in axial view are about $18 \times 12 \mathrm{~cm}$, which was much bigger than the young rhesus monkey, and the estimated skull thickness is $\sim 3 \mathrm{~mm}$, which is also thicker.

Although skull acoustic properties have been measured previous ${ }^{19}$, PA signals may behave differently when they propagate through the skull. To understand the effect of the skull bone on PA signals, we characterized the acoustic properties of the skull bone using PA signals. A 50- $\mu \mathrm{m}$ diameter, 1-cm long human hair was used as the PA source, and the hair was oriented to be parallel to a transducer surface in the detection plane. At the beginning of the measurement, the transducer was used to detect the PA signal from the hair directly. Then the skull bone was put between the transducer and the hair, and the signals were detected after they propagated through the skull. Since the skull bone is not flat, the curvature of the skull bone may introduce uncertainties in incident angles at which the PA pulses strike the skull. To minimize this effect, we oriented the central part of the skull, which is relatively flat, to be parallel to the transducer surface and in the direct path of wave propagation from the PA source to the transducer. During the experiment, the skull was rotated to different angles to provide different incident angles to PA pulses. Two ultrasound transducers used in detecting PA signals have center frequencies at $1 \mathrm{MHz}$ and $2.25 \mathrm{MHz}$ respectively. The monkey 
a)

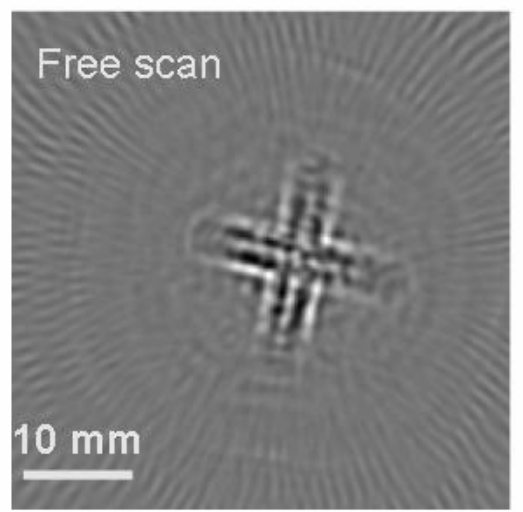

b)

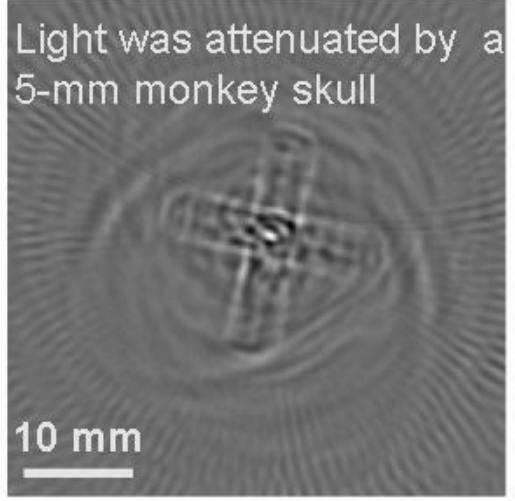

Figure 2 a) PAT of a carbon rod cross: free scan; b) PAT of a carbon rod cross: light was attenuated by a 5-mm thick monkey skull bone.

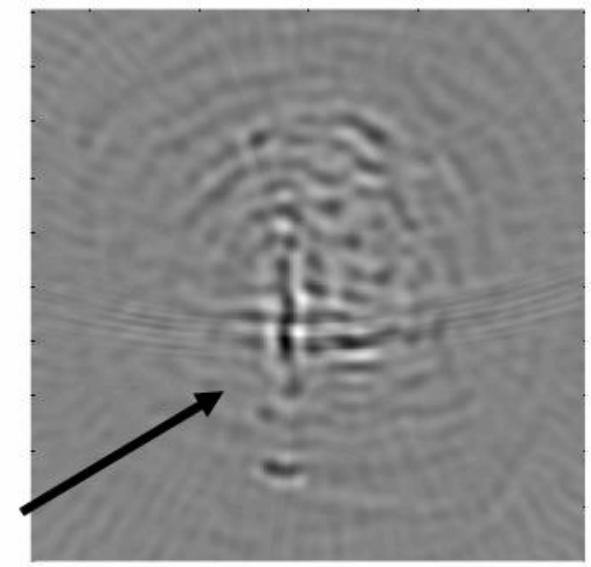

Figure 3 PAT of a carbon rod cross with both light and PA signals attenuated by the skull bone 

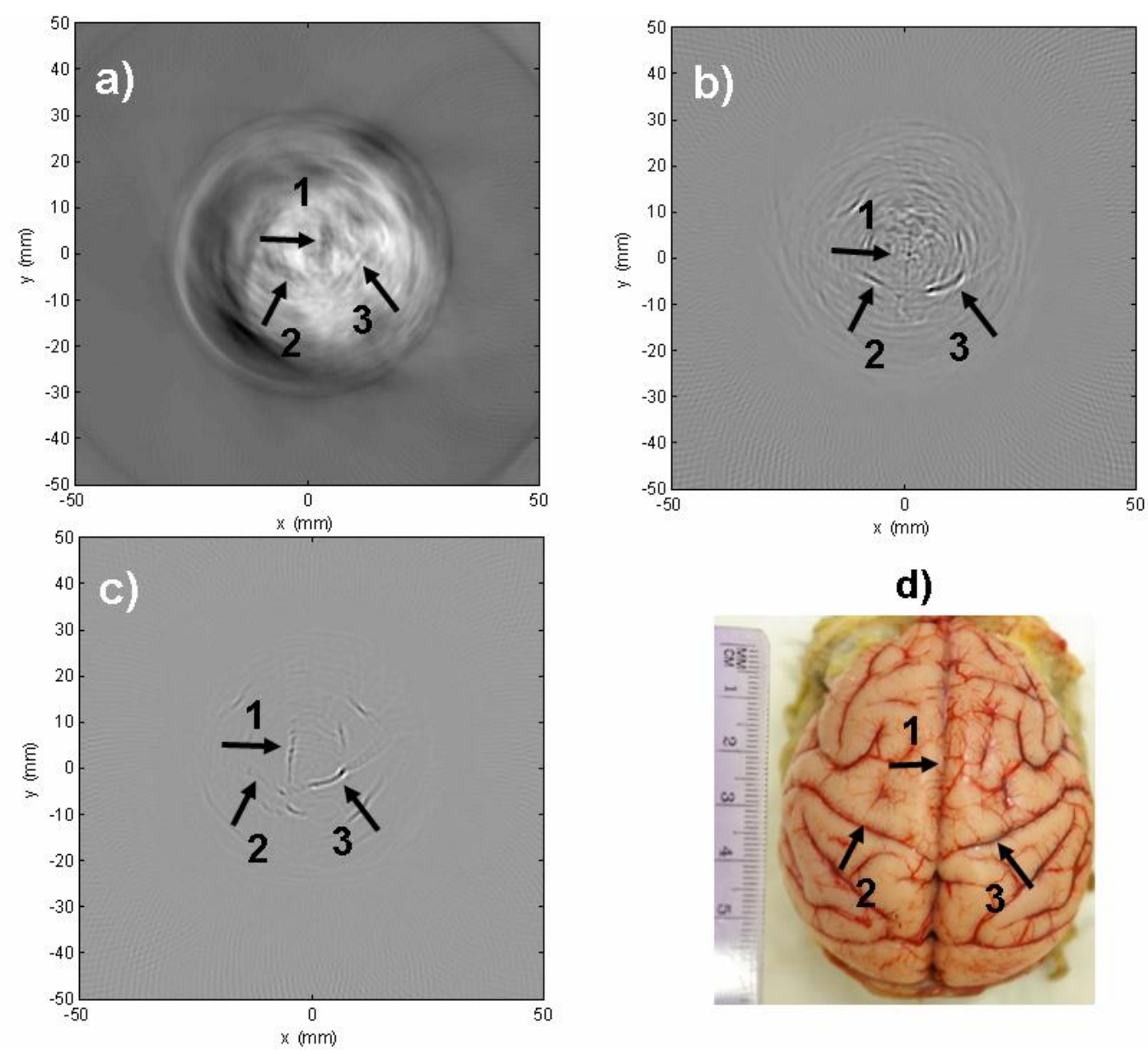

Figure 4 (a) PAT image of a rhesus monkey brain with the intact scalp and skull bone; (b) photograph of the exposed monkey brain; (c) PAT image of the exposed monkey brain; and (d) PAT image of the monkey brain with the skull bone. The blood vessels which can be identified from the PAT image are labeled with numbers on all the images. The same number indicates the same blood vessel

\section{RESULTS}

\subsection{Phantom Results}

The PAT images of the carbon rod cross are shown in Figs. 2 and 3. Fig. 2a shows the result of a free scan, which means there is no skull in place. Fig. $2 \mathrm{~b}$ shows the PAT image after the light was attenuated by the skull bone. Comparing with Fig.2a, Fig $2 \mathrm{~b}$ shows the whole image only with a slightly reduced signal-noise-ratio (SNR). The test confirms that PAT is still possible although the laser was attenuated by a thick skull bone. Fig. 3 shows the PAT image of the carbon rod cross when both light and ultrasound signals have been attenuated. The target is still visible only with slightly distortions.

\subsection{PAT image of monkey brains}


The reconstructed PAT images and the photograph of the exposed brain cortex for the rhesus monkey are shown in Fig. 4. Fig. 4a shows the PAT image of the monkey brain cortex with the intact scalp and skull and Fig. $4 \mathrm{~b}$ with the skull only and Fig. $4 \mathrm{c}$ the exposed brain. Fig. $4 \mathrm{~d}$ is a photograph of the exposed monkey brain. Note that the shape of the brain in Fig. 4d had changed slightly since there was no support of the skull bone, and the dura mater was also removed to expose the brain.

In Fig. 4a, through the intact scalp and skull, six vascular structures can be identified from the PAT image. With the skull only, Fig. 4b shows much more details of the monkey brain cortex than Fig. 4a. Fig. 4c shows the brain cortex with even greater clarity than Fig. $4 \mathrm{~b}$ owing to the lack of the skull effect. Note that since the monkey brain has a curved surface, but our PAT scan in this implementation is 2-dimensional, Figs. 4a-c look quite different from Fig. 4d. However, we can still associate the corresponding blood vessels between them. We labeled the same blood vessels

a)

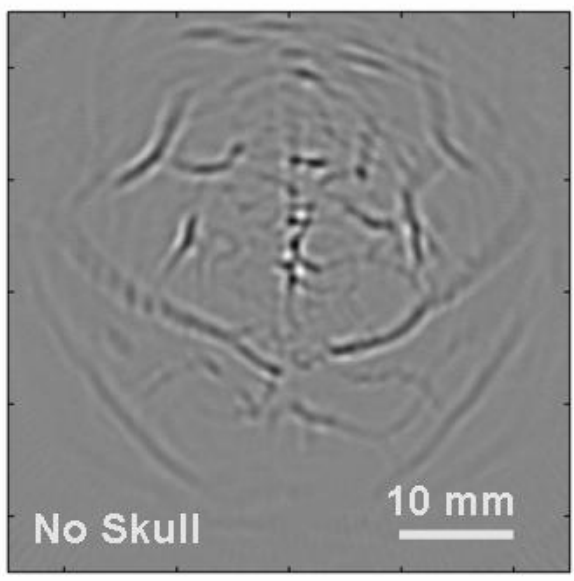

b)

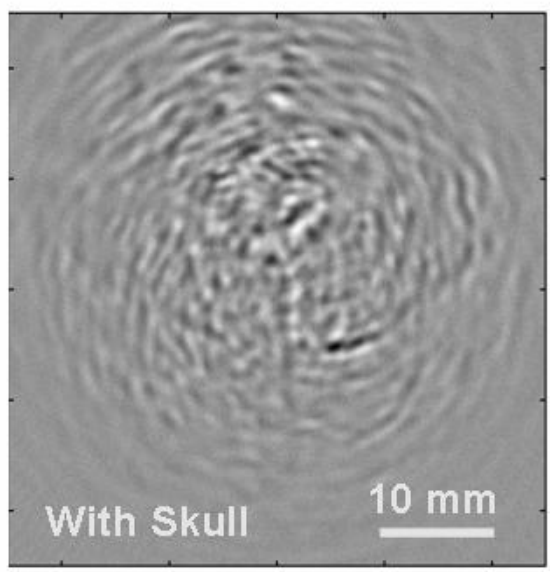

Figure 5 (a) PAT image of the exposed rhesus monkey brain; (b) PAT image of the monkey brain with the skull bone. The $532 \mathrm{~nm}$ laser wavelength was used to obtain these images.

using the same numbers in Figs. 4a-d. The field of view in the PAT image is also smaller than that of the photograph because of the limited laser beam size. Compared with Fig. 4c, Fig. 4a shows similar main vascular structures, which have been labeled with numbers 1-3 on the monkey brain cortex. However, the blood vessels shown in Fig. 4a are thicker than those in Fig. 4c, which was probably caused by the acoustic heterogeneities between the scalp, skull, and dura matter. Again, compared with Fig. 4c, Fig. 4b shows the brain cortex with a slightly reduced contrast due to the skull bone. Therefore, the effect of the skull bone on PAT is insignificant. In fact, the degradation of the image quality due to the scalp is stronger.

Fig. 5 shows the similar results as Fig. 4c-d, but with $532 \mathrm{~nm}$ laser wavelength. Fig. 5a and 5b are the cerebral cortex image with and without the skull, respectively. At $532 \mathrm{~nm}$ wavelength, the blood absorption is high and therefore, Fig. 5a shows more details than Fig. 4c. However, when the skull is on, the image is severely blurred due to the penetration 
depth at $532 \mathrm{~nm}$ wavelength is much shallower than that at $1064 \mathrm{~nm}$ wavelength. This result suggests that $1064 \mathrm{~nm}$ wavelength is more suitable than $532 \mathrm{~nm}$ wavelength for brain cortex imaging through a thick skull bone.

Fig. 6 shows the PAT of the pigtail monkey cerebral cortex. Similar structures as Fig. 4 are identified. We have labeled these structures with the same numbers as in Fig. 4. Compared with Fig. 4a, the field of view in Fig. 6a is smaller. This reduction is due to the size of the skull. The pigtail monkey head used here was much larger than the rhesus monkey head, and the skull attenuation for both light and ultrasound was also bigger. Fig. $6 \mathrm{~b}$ shows an anatomical picture of a pigtail monkey brain, ${ }^{21}$ where a similar structure on the cerebral cortex is shown.

a)

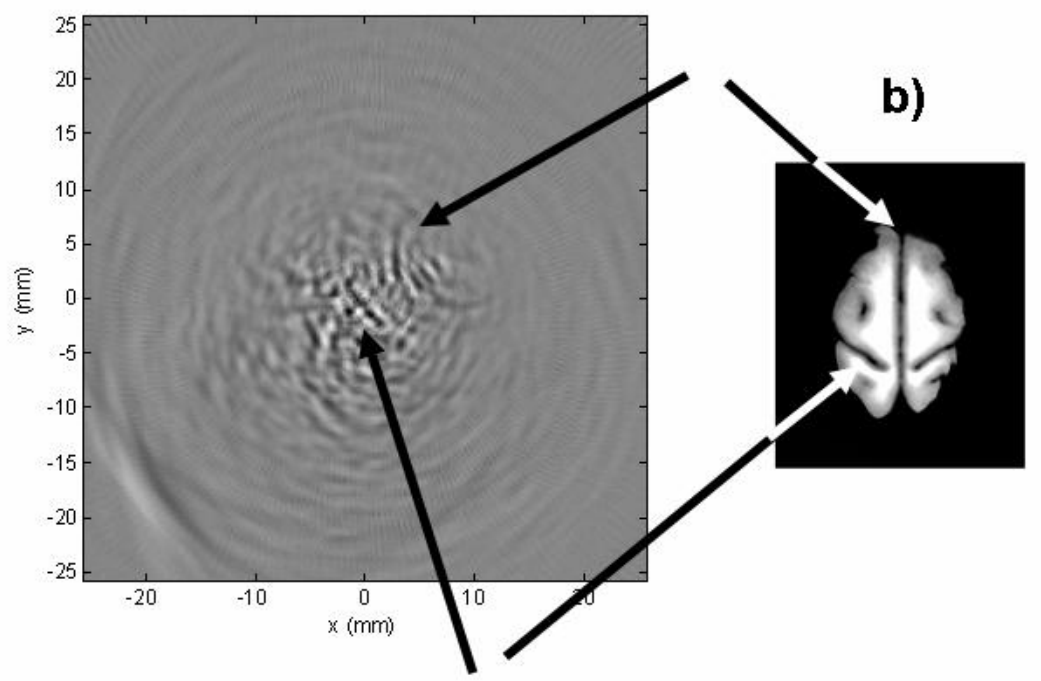

Figure 6 (a) PAT image of a pigtail monkey brain cortex with the intact scalp and skull bone; (b) an anatomical photograph of a pigtail monkey brain.

\subsection{Attenuation versus incident angle}

Fig. 7 shows the attenuation effect of the skull on PA signals. The normalized amplitude of the detected PA signals is shown as a function of the incident angle. As the incident angle increases, the received signal amplitude decreases. After about $35^{\circ}$, the received signal amplitude increases again. This effect may be explained by the generation of shear waves, ${ }^{22}$ which we will discuss in detail next.

\section{DISCUSSION}

We showed that PAT can be applied through a considerably thick skull bone and images can be obtained without corrections to the distortion caused by acoustic heterogeneity. We expect that once the skull gets thicker, the distortion will get severer, and corrections for the distortion may be necessary. 
Besides the skull bone, the degradation on the image quality from Fig. 4c to Fig. 4a can also be explained by the effect of the scalp and dura mater. We do not expect the scalp and dura matter have significant effects on ultrasound signals, but the presence of the scalp and dura matter might severely reduce the penetrated laser energy, and then reduce SNR in the image. In addition, the scalp and dura matter also generate PA signals, which can degrade the quality of brain cortex imaging.

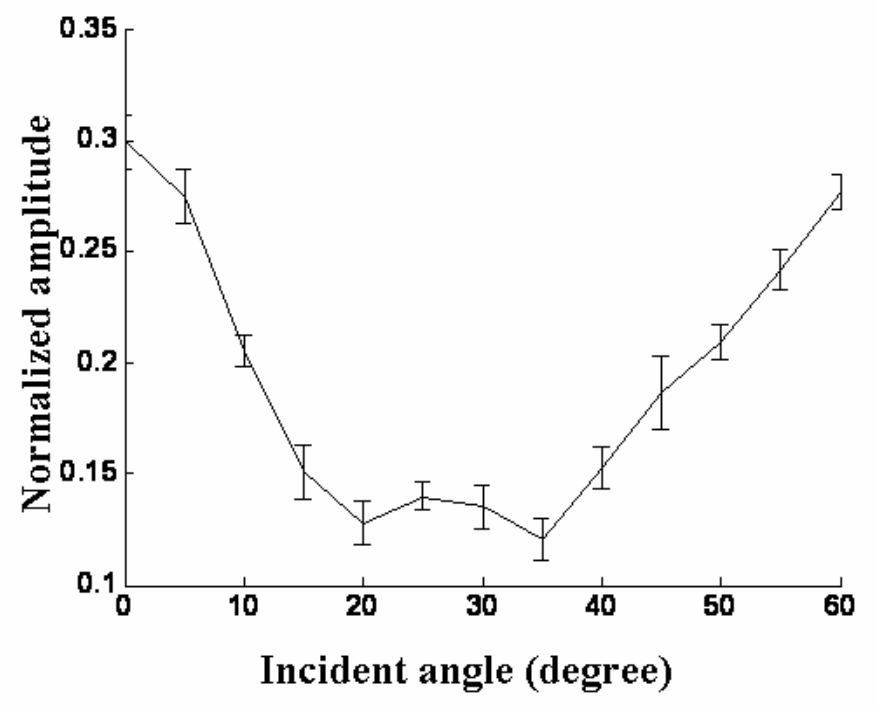

Figure 7 PA signal amplitude detected by a 1-MHz transducer after the signal propagates through a skull bone with different incident angles. All the amplitudes were normalized by the signal amplitude detected when the skull is absent. The error bars shown on the curve are the standard deviation of 10 measurements.

The propagation of ultrasound signals through the monkey skull needs further comments. Since the sound speed of a skull bone is about as twice as that of the soft tissue ${ }^{19}$, the longitudinal wave has a critical incident angle of about $30^{\circ}$ when it propagates from soft tissue to the skull. In other words, once the incident angle is larger than $30^{\circ}$, the longitudinal wave will be totally reflected on the skull surface. In our PAT detection system, we detect PA signals on the horizontal plane of the monkey brain. At this detection location, signals from the blood vessels on brain cortex are very likely to have incident angles larger than $30^{\circ}$ when they propagate towards the skull. Therefore, these signals would be totally reflected at the inner surface of the skull. However, we can still receive ultrasound signals generated from the blood vessels on brain cortex. The explanation is that the signals we detected are from the longitudinal-shearlongitudinal wave conversion. It is well known that at a boundary, the refraction waves consist of the longitudinal waves and shear waves. When the incident angle is beyond the critical angle for the longitudinal waves, the shear waves can still propagate beyond the boundary. If the boundary is a part of a layered material, the shear waves will convert back to longitudinal waves on the other boundary. Through this mechanism, we can still receive signals from blood vessels beyond the critical angle. 


\section{CONCLUSION}

In summary, we have shown that PAT can overcome the effect of a considerably thick skull bone, and the brain cortex imaging of a relative large animal using PAT is feasible. In the future, with the help of PAT contrast agents, it is possible for PAT to image through even thicker skull bones. This result also suggests that PAT may have the potential for human brain cortex imaging.

\section{ACKNOWLEDGEMENTS}

This project is sponsored by National Institutes of Health grants R01 CA106728 and R01 NS46214 (BRP)

\section{REFERENCE}

${ }^{1}$ Schreiber, S. L., Stolz, E. and Valdueza, J. M., "Transcranial ultrasonography of cerebral veins and sinuses," European Journal of Ultrasound, 16, 59-72 (2002).

${ }_{2}^{2}$ Ancora, G., Lanari, M., Lazzarotto, T., Venturi, V., Tridapalli, E., Sandri, F., Menarini, M., Ferretti, E. and Faldella, G.,"Cranial Ultrasound Scanning and Prediction of Outcome in Newborns with Congenital Cytomegalovirus Infection,", The Journal of Pediatrics, 150 (2), 157-162 (2007).

${ }^{3}$ Fry, F., Sanghvi, N., Morris, R., Clendenon, J., Dines, K., Patrick, J. and Goss, S., "Ultrasonic diagnostic system for interactive interrogation of adult brain through intact skull," Invest. Radiol.,vol. 17, 463-469 (1982).

${ }^{4}$ Dines, K., Fry, F., Patrick, J. and Gilmor, R. "Computerized ultrasound tomography of the human head: Experimental results," Ultrason. Imag., vol. 3, 342-351 (1981).

${ }^{5}$ Ylitalo, J., Koivukangas, J. and Oksman, J., "Ultrasonic reflection mode computed tomography through a skullbone," IEEE Trans. Biomed. Eng., vol. 37, 1059-1066 (1990).

${ }^{6}$ White, D. N., Clark, J. M., Chesebrough, J. N., White, M. N. and Campbell, J. K. "Effect of skull in degrading the display of echoencephalographic B and C scans", J. Acoust. Soc. Am., 44, 1339-1345 (1968).

${ }^{7}$ Daverson, S. Evans, D. H. and Bouch, D. C., "The effects of temporal bone on transcranial doppler ultrasound beam shape", Ultrasounds in Med \& Biol., 26 (2), 239-444 (2000).

${ }^{8}$ Smith, S. W., Phillips, D. J., von Ramm, O. T. and Thurstone, F. L., "Some advances in acoustic imaging through the skull,’ Ultrasonic Tissue Characterization II. M. Linzer, ed., NBS Pub. \#525, 209-218 (1979).

${ }^{9}$ Smith, S. W., Chu, K., Idriss, S. F., Ivancevich, N. M., Light, E. D. and Wolf P. D., "Feasibility Study: Real-Time 3-D Ultrasound Imaging Of The Brain", Ultrasound in Med. \& Biol., Vol. 30, No. 10, 1365-1371 (2004).

${ }^{10}$ Ivancevich, N. M., Dahl, J. J., Trahey, G. E. and Smith S. W., "Phase-aberration correction with a 3-D ultrasound scanner: feasibility study," IEEE Trans Ultrason Ferroelectr Freq Control., 53 (8), 1432-1439 (2006).

${ }^{11}$ Tanter, M., Thomas J. L. and Fink, M., "Focusing and steering through absorbing and aberating layers: Application to ultrasonic propagation through the skull," J. Acoust. Soc. Am, 103, 2403-2410 (1998).

${ }^{12}$ Seip, R., VanBaren P. and Ebbini, E., "'Dynamic focusing in ultrasound hyperthermia using implantable hydrophone arrays,' IEEE Trans. Ultrason. Ferroelectr. Freq. Control 41 (5), 706-713 (1994).

${ }^{13}$ Aubry, J. F., Tanter M., Gerber, J., Thomas, J. L. and Fink M., "Optimal focusing by spatio-temporal inverse filter. II. Application to focusing through absorbing and reverberating media", J. Acoust. Soc. Am, 110 (1), 48-58 (2001).

${ }_{15}^{14}$ Xu, M. and Wang, L. V., "Biomedical photoacoustics," Review of Scientific Instruments 77 (4), 041101 (Apr. 2006).

${ }^{15}$ Wang, X., Pang, Y., Ku, G., Xie, X., Stoica, G. and Wang, L. V., "Noninvasive laser-induced photoacoustic tomography for structural and functional imaging of the brain in vivo," Nat. Biotechnol. 21, 803-806 (2003).

${ }^{16}$ Xu., Y. and Wang, L. V., "Rhesus monkey brain imaging through intact skull with thermoacoustic tomography," IEEE Transactions on Ultrasonics Ferroelectrics and Frequency Control 53 (3), 542-548 (2006).

${ }^{17}$ Fry, F. J., "Transkull transmission of an intense focused ultrasonic beam," Ultrasound Med. Biol. 3(2-3), 179-184 (1977).

${ }^{18} \mathrm{Xu}, \mathrm{M} ., \mathrm{Xu}, \mathrm{Y}$. and Wang, L. V., "Time-domain reconstruction algorithms and numerical simulations for thermoacoustic tomography in various geometries," IEEE Biomed. Eng. 50, 1086-1099 (2003).

${ }^{19}$ Fry, F. and Barger, J., “Acoustical properties of the human skull,” J. Acoust. Soc. Am., vol. 63, 1576-1590 (1978). 
${ }^{20}$ Laser Institute of America, American National Standard for Safe Use of Lasers ANSI Z136.1-2000, American National Standards Institute Inc., New York, NY, 2000.

${ }^{21}$ This Photograph was obtained from the monkey atlas, Laboratory of Neuro imaging, UCLA.

${ }^{22}$ Clement, G. T., White, P. J. and Hynynen, K., "Enhanced ultrasound transmission through the human skull using shear mode conversion," J. Acoust. Soc. Am., vol. 115, 1356-1364 (2004). 